\title{
Validez diferencial en la evaluación del constructo frustración
}

\author{
Noelia Rodríguez Sobrino \& Pablo García Medina \\ Departamento de PETRA. Facultad de Psicología \\ Universidad de la Laguna \\ e-mail: noelia.sobrino@terra.es
}

\section{RESUMEN}

En este trabajo se presenta un estudio correlacional que describe la conexión existente entre diferentes estilos de respuesta a la frustración evocada. Para ello nos apoyamos en la Prueba de Respuestas a la Frustración (PRF) y el Cuestionario sobre Valores y Principios (V. y P.), ambos de García-Medina, P. La prueba PRF tiene la finalidad de identificar el constructo de agresividad como respuesta-rasgo. Los restantes estilos de respuesta aislados, según el autor, son el autopunitivo y el solucionador. Se propone para fines de validez diferencial así como de constructo un cuarto factor: frustración expresada. Se aplicó otro cuestionario (Valores y Principios) del que se extrajeron, mediante rotación varimax, dos factores cognitivo-actitudinales. Un primer factor denominado Actitud-disocial y otro segundo denominado Actitud-prosocial. Encontrándose utilidad y apoyo para el primero debido a que presenta validez diferencial significativa al correlacionarlo con los restantes constructos. Se dieron particularmente relaciones positivas entre el estilo de respuesta agresiva y frustración De otro lado, se hallaron relaciones negativas entre un estilo de respuesta de solución y frustración manifestada. La Actitud-disocial, correlaciona positivamente con las respuestas de estilo agresivo. Sin embargo, se obtuvieron relaciones negativas con la tendencia o estilo a dar respuestas de solución a los conflictos. Entendido como respaldo de validez diferencial.

Finalmente, hemos de destacar que la actitud prosocial se relacionó de forma positiva con la respuesta autopunitiva en un subgrupo de casos que presentaban menor tolerancia a la frustración.

En relación a las pruebas se deduce a partir de los resultados que el PRF se muestra más sensible que el cuestionario $\mathrm{V}$ y $\mathrm{P}$ basado en dos factores $\mathrm{y}$ esta sensibilidad es mayor en personas que puntúan bajo en el estilo de respuesta agresiva.

Palabras Clave: Respuesta a la frustración, actitud disocial. 


\title{
Differential validity in the evaluation of the construct frustration
}

\begin{abstract}
In this work, we present the results of a correctional study that catch and describes the probable connection between different styles of response evoked frustration. For it we rest in Responses to Frustration test (PRF) and Questionnaire about values and principles (V\&P) both by García-Medina, P. The PRF test has between others, the purpose of catching the construct of aggression as an answer-feature. The remaining styles of response isolated, according to the author are, self-punishing and solving. One proposes for ends of differential validity as well as construct validity a fourth factor: expressed frustration. There was applied another questionnaire (Values and Principles) from that two factors were extracted, by means of rotation varimax, cognitive - actitudinales. The component named antisocial attitude and the component named prosocial attitude. Being utility and support for the first one, which presents differential significant validity on having correlated it with the remaining constructs. We find positive relations between aggressive response and frustration. In the same way, we find negative relations between solving response and expressed frustration. The antisocial attitude, as it was evaluated proved to be correlated positively with the aggressive answers. Understood these, directed well to others or directed one itself. They converge logic and semantically with a high degree of frustration. Nevertheless, negative relations were obtained by the trend or style to giving answers of solution to the conflicts raised in the exercise book of proof. Understood as support of the differential validity.

Finally, we have to stand out that the prosocial conduct related positive with self-punishing in the cases that were presenting less tolerance to the frustration.

In relation to the tests there is inferred of the results that the PRF proves to be more sensitive to catch the trend actitudinal that the questionnaire V\&P based on two factors and this sensibility it is high in persons who count low in the style of aggressive response.
\end{abstract}

Keywords: Response frustration, antisocial attitude. 


\section{Introducción - Marco Teórico}

\section{El estilo de respuesta}

Según García-Medina (1991) las personas ante una misma situación pueden manifestar diferentes estilos de respuesta cuando se les enfrenta a diversos conflictos. Es decir, pueden dar diferentes tipos de respuesta manifestadas al menos sobre protocolos o cuestionarios. Citando a este autor, ante un mismo hecho percibido como impedimento para el logro de una meta, podemos articular una actitud de tono agresivo, que no pasa más allá de ser un componente agresivo, o pasar directamente a responder con violencia. En este trabajo denominamos a esta tendencia, en el marco de un modelo dimensional, "estilo de respuesta agresiva". Por otra parte, podemos dirigir la violencia hacia nosotros mismos, lo que denominaremos "respuesta autopunitiva", así como podemos resolver el conflicto planteado de manera pacificadora, la que aquí denominamos "respuesta resolutiva o solucionadora".

\section{El tipo de actitud}

En el contexto de este trabajo la Actitud disocial se define como el posicionamiento mental de enfrentamiento contra los mandatos de las figuras que representan autoridad, así como la idea de trasgresión anticipada de las normas sociales e institucionales.

Por contra, la Actitud prosocial se considera al conjunto de razonamientos que pueden acompañar a las conductas que los seres humanos realizan de forma voluntaria en favor de otros, con independencia de que la mayor parte de los casos pueda o deba revertir en beneficio propio. Como sostiene Batson (1998) no tiene por qué darse una necesaria correspondencia entre conducta prosocial y altruismo.

\section{La Frustración}

La propuesta de Dollard, Doob, Miller, Mower y Sears (1939), sostiene que toda agresión está necesariamente provocada por una frustración. Dicha interpretación, al principio incluye la no consecución de una meta como productora de agresividad y a partir de ahí se evoluciona hasta la frustración como suspensión de una recompensa. Los 
autores dicen que se provoca la aparición de un proceso de cólera que, cuando alcanza un grado determinado, puede producir la agresión física o verbal.

Consumir el acto de agresión serviría por lo tanto para eliminar la frustración sentida de modo que la persona logra su equilibrio, se estabilizará, hasta que concurra una nueva fuente externa de frustración (Dollard et al, 1939). Según esta hipótesis, la agresión es la respuesta natural predominante ante un bloqueo o sentimiento de frustración y, se afirma que, la frustración activa un impulso agresivo que sólo se reduce mediante alguna forma de respuesta agresiva. Se trata en esencia, de un modelo explicativo dicotómico.

Apoyando tal idea pero dando opción a una versión multifactorial al tiempo que dimensional García-Medina (1991), argumenta que se pueden proponer tres tipos o estilo: agresión, autopunición y resolución del conflicto. Los que pueden ir acompañados o no de un sentimiento desagradable, tal sentimiento sería el de frustración. García-Medina, Bethencourt, Ramos y González (1996), proponen entender el constructo de la frustración como un factor independiente y no necesario en las situaciones que pueden provocar una actitud agresiva. El factor desagrado evaluado en el cuestionario PRF (García-Medina, 1992) es visto en este contexto como un indicador de frustración, que puede ser independiente a su vez de los tres estilos de propuestos, que además puede actuar como un modulador de los estilos de respuesta mencionados.

\section{Objetivos}

El presente trabajo de investigación tiene por objetivo principal dar respaldo a la relación que hemos encontrado entre la actitud de tipo antisocial y la frustración, en base a los diferentes tipos de respuesta que se proponen:

a) Respuesta agresiva

b) Respuesta autopunitiva

c) Respuesta solucionadora

Se trata en esta fase de estudio, de aislar componentes cognitivo-actitudinales que hacen referencia a predisponentes de actuación unas veces de tipo prosocial y otras de tipo antisocial. Se toma para ello a puntuadores bajos y altos en frustración.

Revista Española de Investigación Criminológica

Artículo 5, Número 7 (2009) $\quad$ www.criminología.net

ISSN: $1696-9219$ 


\section{Método}

\section{Participantes}

La muestra total de este estudio consta de 91 voluntarios escogidos al azar de ambos sexos, residentes en la Comunidad Autónoma de Canarias. La edad mínima de participación es de 12 años y mayores de 40 años. Las variables de la muestra incluyen sexo, edad y nivel de estudios se reflejan en los cuadros siguientes.

\begin{tabular}{|c|c|c|}
\hline Edad & & \\
& Frecuencia & Porcentaje \\
\hline 12 a 17 & 4 & 4,4 \\
18 a 25 & 26 & 28,6 \\
26 a 40 & 21 & 23,1 \\
$>40$ & 40 & 44,0 \\
\hline Total & 91 & 100,0 \\
\hline
\end{tabular}

\begin{tabular}{|l|c|c|}
\hline Sexo & Frecuencia & Porcentaje \\
\hline Hombre & 41 & 45,1 \\
Mujer & 50 & 54,9 \\
\hline Total & 91 & 100,0 \\
\hline
\end{tabular}

\begin{tabular}{|l|c|c|}
\hline Nivel de estudios & Frecuencia & Porcentaje \\
\hline No Responde & 3 & 3,3 \\
Sin estudios & 13 & 14,3 \\
Con estudios básicos obligatorios (E.G.B. / 4 ${ }^{\text {o }}$ E.S.O.) & 24 & 26,4 \\
Con estudios medios (B.U.P y C.O.U) & 26 & 28,6 \\
Con estudios superiores (Diplomado / Licenciado) & 25 & 27,5 \\
\hline Total & 91 & 100,0 \\
\hline
\end{tabular}




\section{Materiales}

En la investigación se han utilizado los siguientes instrumentos:

1. Cuestionario sobre Valores y Principios (V. y P.) García-Medina, P. (1993). Doc. interno. Dep. P.E.T.P. ULL.

2. Prueba de Respuestas a la Frustración (PRF) García-Medina. P. (1992).

Vers. Exp. Dep. P.E.T.P. ULL. (sin publicar)

\section{Procedimiento}

Mediante el pase de cuestionarios se recabaron los datos, contabilizando un total de 91 pruebas contestadas. El pase de cuestionarios se realizó aleatoriamente a voluntarios/as. A todas las personas se les garantizó el anonimato de sus respuestas. Así mismo se les explicó cómo cumplimentar las pruebas. Los datos recopilados fueron introducidos en ordenador y procesados utilizando el paquete estadístico SPSS.

\section{Resultados}

A fin de conocer la fiabilidad, mediante el índice de consistencia interna $\alpha$ de Cronbach, se obtuvieron los resultados que se observan en la tabla1.

Tabla 1. Índice fiabilidad de los factores de actitud tipo: Prosocial - Antisocial

\begin{tabular}{|c|c|c|c|}
\hline & Escala Total & $\begin{array}{l}\text { Factor } 1 \\
\text { Actitud Prosocial }\end{array}$ & $\begin{array}{l}\text { Factor } 2 \\
\text { Actitud Antisocial }\end{array}$ \\
\hline Número de casos & 91 & 91 & 91 \\
\hline Número de ítems & 23 & 12 & 11 \\
\hline Alfa de Cronbach $(\alpha)$ & 0,71 & 0,69 & 0,68 \\
\hline
\end{tabular}

A continuación se presenta, a modo de ejemplo, algunos de los ítems que componen cada uno de los factores ${ }^{1}$ :

\footnotetext{
${ }^{1}$ La matriz de componentes puede verse en el Anexo I.
}

Revista Española de Investigación Criminológica Artículo 5, Número 7 (2009) $\quad$ www.criminología.net

ISSN: $1696-9219$ 
- Factor 1: "Actitud Prosocial"

$\rightarrow \quad$ It. 8.- Suelo aceptar las decisiones de mis familiares de más edad

$\rightarrow \quad$ It. 53.- Me cuesta muy poco llevar a cabo las sugerencias hechas por cualquier agente de la autoridad

$\rightarrow \quad$ It. 54.- Me cuesta muy poco cumplir con las normativas legales en líneas generales

$\rightarrow \quad$ It. 55.- Soy partidario/a de que en ningún caso nos saltemos las normas impuestas legalmente

$\rightarrow \quad$ It. 65.- Cuando una ley es buena para los demás, aunque me perjudique, la acepto de buena gana

- Factor 2: "Actitud Antisocial”

$\rightarrow \quad$ It. 6.- Si no hay más remedio, es más eficaz hacerse respetar a la fuerza, que esperar a lo que dirá un juez

$\rightarrow \quad$ It. 11.- Para llegar antes, si tengo que cruzar un paso de peatones en rojo, lo hago

$\rightarrow \quad$ It. 12.- Si no me justifican por qué no debo hacer alguna cosa termino haciéndola

$\rightarrow \quad$ It. 13.- Considero normal que, a un/a hijo/a, se le de un cachete para hacerle entrar en razón

$\rightarrow \quad$ It. 18.- Si no me justifican la existencia de una norma me rebelo y enfurezco

Como se menciona en los objetivos, en el presente estudio interesa conocer la existencia o no de relación entre los diferentes estilos de respuesta, la variable frustración y los dos factores extraídos anteriormente, denominados actitud prosocial y actitud antisocial. Para ello en las siguientes páginas se exponen las tablas de las correlaciones obtenidas.

Las correlaciones encontradas, para toda la muestra 91 casos, entre los estilos de respuesta y los diferentes tipos de actitud se presentan en la tabla 2. 
Tabla 2. Correlaciones entre estilos de respuesta, tendencia actitudinal y desagradofrustración. $\mathrm{N}=91$

\begin{tabular}{|l|c|c|c|c|c|c|}
\hline & $\begin{array}{c}\text { R. } \\
\text { Agresiva }\end{array}$ & $\begin{array}{c}\text { R. } \\
\text { Autopunitiva }\end{array}$ & $\begin{array}{c}\text { R. } \\
\text { Solucionadora }\end{array}$ & $\begin{array}{c}\text { Desagrado- } \\
\text { Frustración }\end{array}$ & $\begin{array}{c}\text { Act. } \\
\text { Antisocial }\end{array}$ & $\begin{array}{c}\text { Act. } \\
\text { Prosocial }\end{array}$ \\
\hline R. Agresiva & &, 14 &,$- 87(* *)$ &, $47(* *)$ &, $49(* *)$ &,- 10 \\
\hline R. Autopunitiva & & &,$- 61(* *)$ &,- 09 &, $29(* *)$ &, 12 \\
\hline R. Solucionadora & & & &,$- 33(* *)$ &,$- 53(* *)$ &, 02 \\
\hline $\begin{array}{l}\text { Desagrado- } \\
\text { Frustración }\end{array}$ & & & & &, $30(* *)$ &, 12 \\
\hline
\end{tabular}

** La correlación es significativa al nivel 0,01 (bilateral)

Como se observa en la tabla 2, la respuesta de tendencia agresiva, bien sea hacia uno mismo "respuesta autopunitiva" bien sea hacia otros "respuesta agresiva" correlaciona de manera significativa y positiva con la actitud de tipo antisocial, (.49) y (.29) respectivamente.

Existe también una relación positiva entre la tendencia de respuesta agresiva y frustración (.47), la cual no se observa cuando el estilo de dicha agresividad es dirigida hacia uno mismo. Sin embargo, se aprecia una relación entre ésta variable y la tendencia de respuesta solucionadora, aunque es negativa (-.33).

Por otro lado, se encuentran relaciones negativas entre la tendencia a resolver situaciones de modo pacificador "respuesta solucionadora o resolutiva" y la actitud de tipo antisocial (-.53).

Este tipo de actitud correlaciona de forma significativa y positiva con la variable frustración (.30).

Finalmente cabe destacar que no se encuentran relaciones entre la actitud de tipo prosocial y alguno de los estilos de respuesta así como tampoco con la variable frustración.

Dado que la presente investigación se centra en conocer las características de los más agresivos, en las siguientes páginas se muestran las relaciones halladas para este grupo de sujetos y las variables objeto de estudio.

Tras seleccionar del total de la muestra, los casos en función de las mayores puntuaciones en tendencia agresiva, el grupo de voluntarios objeto de análisis se concreta en 20 casos. Los resultados obtenidos se muestran en la tabla 3.

Revista Española de Investigación Criminológica

Artículo 5, Número 7 (2009) $\quad$ www.criminología.net

ISSN: 1696-9219 
Tabla 3. Correlaciones entre estilos de respuesta, tendencia actitudinal y desagradofrustración de los puntuadores altos en respuesta estilo agresivo. $\mathbf{N}=\mathbf{2 0}$

\begin{tabular}{|l|c|c|c|c|c|c|}
\hline & $\begin{array}{c}\text { R. } \\
\text { Agresiva }\end{array}$ & $\begin{array}{c}\text { R. } \\
\text { Autopunitiva }\end{array}$ & $\begin{array}{c}\text { R. } \\
\text { Solucionadora }\end{array}$ & $\begin{array}{c}\text { Desagrado- } \\
\text { Frustración }\end{array}$ & $\begin{array}{c}\text { Act. } \\
\text { Antisocial }\end{array}$ & $\begin{array}{c}\text { Act. } \\
\text { Prosoc. }\end{array}$ \\
\hline R. Agresiva & &,- 17 &,$- 51\left(^{*}\right)$ &, 30 &, 32 &,- 13 \\
\hline R. Autopunitiva & & &,$- 77\left(^{* *}\right)$ &,- 42 &, 10 &,- 12 \\
\hline $\begin{array}{l}\text { R. } \\
\text { Solucionadora }\end{array}$ & & & &, 17 &,- 30 &, 19 \\
\hline $\begin{array}{l}\text { Desagrado- } \\
\text { Frustración }\end{array}$ & & & & &, $56\left(^{*}\right)$ &, 17 \\
\hline
\end{tabular}

* La correlación es significante al nivel 0,05 (bilateral)

** La correlación es significativa al nivel 0,01 (bilateral)

Como se observa, al seleccionar únicamente a los puntuadores altos en estilo de respuesta agresiva, las relaciones encontradas varían respecto al total de la muestra (tabla 2). En esta ocasión, no existen correlaciones entre la actitud y alguno de los estilos de respuesta, aunque sí se aprecia una relación significativa y positiva entre la actitud antisocial y la variable frustración (.56). Fíjese que esta relación es mayor que la encontrada para la muestra total (.30) (tabla 2).

A fin de establecer diferencias teniendo en consideración la tendencia agresiva, a continuación se han seleccionado los casos que correspondían con puntuadores bajos en estilo de respuesta agresividad, considerándolos, por tanto, como los menos agresivos/as o con menor tendencia a la agresividad, éstos se concretaron en 53 casos. Los resultados se muestran en la tabla 4.

Tabla 4. Correlaciones entre estilos de respuesta, tendencia actitudinal y desagradofrustración de los puntuadores bajos en respuesta estilo agresivo. $\mathbf{N}=53$

\begin{tabular}{|l|r|r|r|r|r|r|}
\hline & $\begin{array}{c}\text { R. } \\
\text { Agresiva }\end{array}$ & $\begin{array}{c}\text { R. } \\
\text { Autopunitiva }\end{array}$ & $\begin{array}{c}\text { R. } \\
\text { Solucionadora }\end{array}$ & $\begin{array}{c}\text { Desagrado- } \\
\text { Frustración }\end{array}$ & $\begin{array}{c}\text { Act. } \\
\text { Antisocial }\end{array}$ & $\begin{array}{c}\text { Act. } \\
\text { Prosocial }\end{array}$ \\
\hline R. Agresiva & &, 15 &,$- 67(* *)$ &, $45(* *)$ &, 16 &, 03 \\
\hline R. Autopunitiva & & &,$- 83(* *)$ &,- 04 &, $39(* *)$ &, 26 \\
\hline R. Solucionadora & & & &,- 22 &,$- 38(* *)$ &,- 21 \\
\hline $\begin{array}{l}\text { Desagrado- } \\
\text { Frustración }\end{array}$ & & & & &, 04 &, 15 \\
\hline
\end{tabular}

* La correlación es significativa al nivel 0,01 (bilateral)

Revista Española de Investigación Criminológica

Artículo 5, Número 7 (2009) $\quad$ www.criminología.net

ISSN: $1696-9219$ 
Tal y como se observa en la tabla, aparecen correlaciones significativas para la actitud de tipo antisocial. Ésta se relaciona de forma significativa y positiva con la tendencia a agredirse a uno mismo (.39) y de forma también significativa pero negativamente con una respuesta de estilo resolutivo (-.38).

Por otro lado, se observa la relación positiva y significativa entre la variable frustración y el estilo de respuesta agresiva hacia los demás (.45).

Señalar que, de nuevo la actitud antisocial no se relaciona con ninguna de las variables objeto de estudio.

Es de interés en el presente trabajo obtener información sobre el sentimiento de desagrado que muestran las personas de esta población en una situación concreta, entendido aquí como frustración. Por ello se han seleccionado en primer lugar a las personas que manifiestan niveles más altos de desagrado o lo que aquí se entiende como mayor sentimiento de frustración. La muestra se conforma en 42 casos y los resultados se muestran en la tabla 5 .

Tabla 5. Correlaciones entre estilos de respuesta, tendencia actitudinal y desagradofrustración en los puntuadores altos en frustración. $\mathrm{N}=42$

\begin{tabular}{|l|c|c|c|c|c|c|}
\hline & $\begin{array}{c}\text { R. } \\
\text { Agresiva }\end{array}$ & $\begin{array}{c}\text { R. } \\
\text { Auto- } \\
\text { punitiva }\end{array}$ & $\begin{array}{c}\text { R. } \\
\text { Soluciona- } \\
\text { dora }\end{array}$ & $\begin{array}{c}\text { Desagrado } \\
\text { / Frustra- } \\
\text { ción }\end{array}$ & $\begin{array}{c}\text { Act. } \\
\text { Anti- } \\
\text { social }\end{array}$ & $\begin{array}{c}\text { Act. } \\
\text { Pro- } \\
\text { social }\end{array}$ \\
\hline R. Agresiva & &,- 04 &,$- 81(* *)$ &, 06 &, $63(* *)$ &,- 18 \\
\hline R. Autopunitiva & & &,$- 55(* *)$ &,- 20 &, 30 &, $33(*)$ \\
\hline R. Solucionadora & & & &, 07 &,$- 70(* *)$ &,- 04 \\
\hline $\begin{array}{l}\text { Desagrado- } \\
\text { Frustración }\end{array}$ & & & & &, 01 &, 02 \\
\hline
\end{tabular}

** La correlación es significativa al nivel 0,01 (bilateral)

* La correlación es significante al nivel 0,05 (bilateral)

Como se observa, al extraer de la muestra total concretamente a puntuadores altos en sentimiento de desagrado-frustración, se establecen nuevas relaciones.

En esta ocasión la actitud de tipo prosocial se relaciona de forma significativa y positivamente con un estilo de respuesta autopunitiva (.33). 
Por otro lado, la actitud de tipo antisocial se relaciona significativamente y de forma positiva con el estilo de respuesta agresiva hacia los demás (.63) y de forma negativa con la tendencia a resolver la situación de manera pacificadora (-.70).

Veamos ahora qué resultados se obtienen al conformar la población con los puntuadores bajos en la variable frustración, siendo éstos 39 los casos seleccionados, en la tabla 6 se muestran los datos hallados.

Tabla 6. Correlaciones entre estilos de respuesta, tendencia actitudinal y desagradofrustración en los puntuadores bajos en frustración. $\mathrm{N}=39$

\begin{tabular}{|l|c|c|c|c|c|c|}
\hline & $\begin{array}{c}\text { R. } \\
\text { Agresiva }\end{array}$ & $\begin{array}{c}\text { R. } \\
\text { Autopunitiva }\end{array}$ & $\begin{array}{c}\text { Respuesta } \\
\text { Solucionadora }\end{array}$ & $\begin{array}{c}\text { Desagrado- } \\
\text { Frustración }\end{array}$ & $\begin{array}{c}\text { Actitud } \\
\text { Antisocial }\end{array}$ & $\begin{array}{c}\text { Actitud } \\
\text { Prosocial }\end{array}$ \\
\hline R. Agresiva & &, $44(* *)$ &,$- 92(* *)$ &, $44(* *)$ &, 19 &,- 10 \\
\hline R. Autopunitiva & & &,$- 75(* *)$ &,- 04 &, $34(*)$ &,- 23 \\
\hline R. Solucionadora & & & &,- 30 &,- 29 &, 18 \\
\hline $\begin{array}{l}\text { Desagrado- } \\
\text { Frustración }\end{array}$ & & & & &, 07 &, 17 \\
\hline
\end{tabular}

** La correlación es significativa al nivel 0,01 (bilateral)

* La correlación es significante al nivel 0,05 (bilateral)

Tal y como se refleja en la tabla 6 las relaciones han variado en relación al grupo anterior (tabla 5), para éstos se observa una relación significativa y positiva entre la actitud de tipo antisocial y la tendencia de respuesta autopunitiva o agresiva hacia uno/a mismo/a (.34).

Así mismo se establece relación significativa y positiva en el estilo de respuesta agresiva y el sentimiento de desagrado-frustración (.44).

Contrariamente, no se han hallado relaciones significativas entre la actitud de tipo prosocial y la variable frustración o alguno de los estilos de respuesta. 


\section{Discusión}

En principio se puede señalar que del cuestionario Valores y Principios (GarcíaMedina, 1993) se han extraído dos componentes, actitud prosocial y actitud antisocial, de los cuales sólo uno de ellos, la actitud de tipo antisocial, actúa con aceptable validez diferencial.

En este trabajo se ha encontrado que la variable que denominamos frustración se relaciona de forma significativa y positiva, únicamente con el estilo de respuesta agresiva, haciéndolo de forma negativa con el estilo de respuesta solucionador.

Por tanto no se obtiene un apoyo empírico, en esta investigación a los resultados encontrados por García-Medina y cols. (1996) quienes proponer entender el constructo de la frustración como un factor independiente y no necesario de las situaciones que puede provocar una actitud agresiva.

Así, en base a los resultados obtenidos se tiende a apoyar la hipótesis de vincular el comportamiento agresivo con una experiencia frustrante tal como proponían Dollard y cols. (1939) en su teoría de la frustración-agresión, tras todo acto agresivo se encontraría una frustración o en términos generales, toda frustración conllevaría a un comportamiento agresivo. 


\section{Bibliografía}

Batson, C.D. (1998). Altruism and Prosocial behavior. En D.T. Gilbert, S. Fiske y G. Lindzey (Eds.). The handbook of social psychology (4a ed., Vol. II, pp. 282-316). Nueva York: McGraw-Hill

Dollard, J., Doob, L., Miller, N.E., Mower, H.O. \& Sears, R.R. (1939). Frustration and aggression. New Haven: Yale Un. Press

García-Medina, P., Ramos, C. y Bethencourt, J.M. (1991). Agresión y delincuencia: Respuestas a situaciones o Patrón de Conductas establecido. (Dpto. P.E.T.P. La Laguna). (Pryto. Sub. Conserjería de Educación y Deportes. Gobierno de Canarias).

García-Medina, P., Bethencourt, J.M., Ramos, C. \& González, M.E. (1996). "Frustración - Agresión: No linealidad de una teoría". En E. Sola Reche, J.M. Bethencourt Pérez, P. Matud Aznar y P. García-Medina: Implicaciones de la Psicología en la Criminología Actual .Granada: Ed. Comares. 


\section{ANEXO I}

El interés se centra en realizar un análisis factorial de 23 elementos extraídos del cuestionario V y $\mathrm{P}$ para poder denominar semánticamente a los factores, en función de los ítems que los conformen.

Previo a dicho análisis se lleva a cabo la prueba de Kaiser Meyer Olkin, para aceptar el método factorial como posibilidad de análisis de los ítems.

\section{KMO y prueba de Bartlett}

\begin{tabular}{|ll|l|}
\hline Kaiser-Meyer-Olkin. &, 56 \\
\hline Prueba de Bartlett & Chi-cuadrado aprox. & 511,04 \\
& gl & 253 \\
& Sig. &, 00 \\
\hline
\end{tabular}




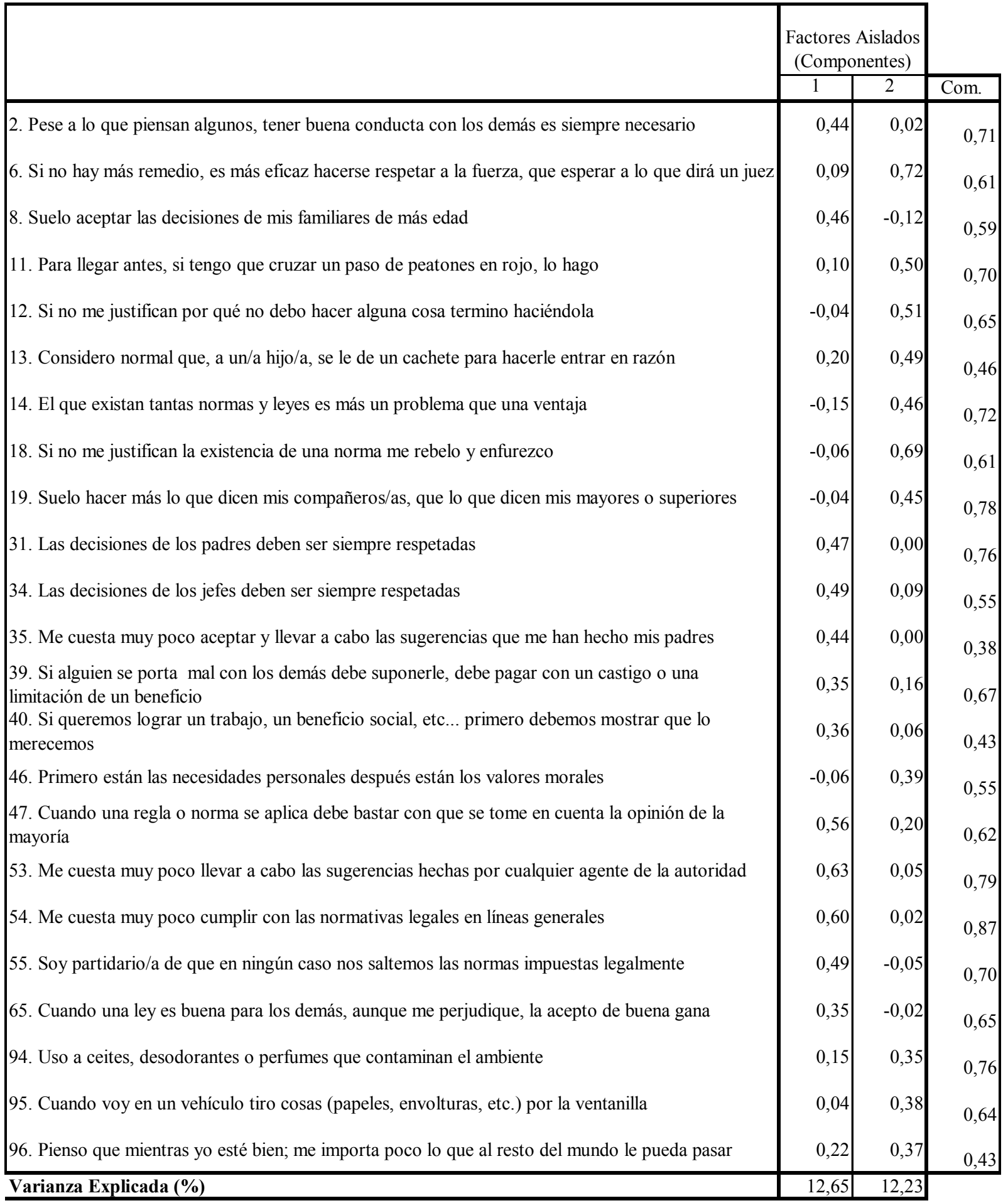

Tabla 1. Método de extracción: Análisis de componentes principales.

Método de rotación: Normalización Varimax con Kaiser.

a. La rotación ha convergido en 3 iteraciones.

* Contenidos: Factor 1 = Tipo Antisocial; Factor 2 = Tipo Prosocial 